\begin{tabular}{c} 
International Journal of Engineering \& Technology, 7 (3) (2018) 1648-1655 \\
International Journal of Engineering \& Technology \\
SPC \\
Website: $\begin{array}{c}\text { ww. sciencepubco.com/index.php/IJET } \\
\text { doi: } 10.14419 / \text { ijet.v7i3.14618 } \\
\text { Research paper }\end{array}$ \\
\hline
\end{tabular}

\title{
Spectral analysis for evaluation of the optical fiber cable performance using experimental and ANN techniques
}

\author{
H. J. Abbas * \\ Telafer University, Department of Scientific Affairs, Iraq \\ *Corresponding author E-mail: dr.hussain@uotelafer.edu.iq
}

\begin{abstract}
This work deals with the performance evaluation of the optical fiber cables by calculating the changes in the power spectral density, power spectrum, and phase of the response signals from which the faults can be deducted and identified and accordingly the performance can be evaluated. An experimental program has been conducted by exciting the cable and then measuring the power spectral density, power spectrum, and phase of the response signal for the faulty and un-faulty cables. The calculated and measured results are fed to the ANN system in order to train the ANN program, then the trained ANN package will be used as a parametric study of deduction and identification of cable faults. The ANN system detects and identities the faults in optical fiber. The experimental results power spectral density, power spectrum, and phase measurements are compared with ANN techniques results. Both results showed a good agreement with maximum error less than (1.26\%). Finally, the paper shown with vibration test for cable and evaluate for electrical characterizations can be prediction for crack depth or location parameters.
\end{abstract}

Keywords: Detection; Identification; Fault; Power Spectral Density; Power Spectrum; Phase.

\section{Introduction}

There are different techniques to test and evaluate the performance of the fiber optical cable and links. According to these evaluation tests, it could be possible to detect, locate and discriminate the faults on the cable. The faults that occur on the cable deteriorate the performance of the fiber optic cables. Therefore the detection, location and identification of these faults are very importation in order to facilitate the treatment of them and recover the performance of the cable.

There are three techniques used to detect the faults in the optical fiber cable. These techniques include optical time domain reflectometry OTDR, low coherence frequency domain reflectometry (OLCR) and coherence optical frequency domain reflectometry (OFDR), [1-3], all these techniques depend on the measurement of the attention and loss of the transmitted signal a long the fiber optical cable. Jihong Greng et al. [4], have developed long range coherent frequency domain reflectometry for measurement of the back scattering and Fresnel reflection.

The faults in the optical fiber cables according to results of the measurement using the above techniques are detected, located and identified. The main disadvantages of the above technique is the dependency on the weak reflected signals which need very high resolution and sensitivity equipment's. Hussain J. Abbas et al. [5] have developed a new technique for the testing of the cables for the detection, location and identification of the faults based on the frequency response of the existing signal of the optical cable. There, in General the effect of crack in engineering structure are investigated by many researchers, as presented in [6-7], for various structures such as beam, plate, shell, and cable with static and dynamic behaviors. Also, the crack defect leads to increasing the frequency response of structure under excitation.

In this work a low frequency signal is used to excite the optical cable and response of the excitation signal is detected and analysed to determine the frequencies of the response of exited cable. Fourier transform equation is used to calculate the frequency spectrum of the response of the structure as follows, [8],

$X(f)=\int_{-\infty}^{+\infty} x(t) e^{-j 2 \pi f t} d t$

Where $\mathrm{X}(\mathrm{f})$ is the frequency spectrum of exited cable. $\mathrm{x}(\mathrm{t})$ is the excitation signal.

The frequency spectrum of the response signal contains the magnitude and phase spectrum as follows,

$$
\begin{aligned}
& |\mathrm{X}(\mathrm{f})|=\sqrt{\mathrm{R}^{2}+\mathrm{I}^{2}} \\
& <\mathrm{X}(\mathrm{f})=-\tan ^{-1} \frac{\mathrm{I}}{\mathrm{R}}
\end{aligned}
$$

Where, $|\mathrm{X}(\mathrm{f})|=$ magnitude spectrum, $\mathrm{R}=$ real part of the spectrum, $\mathrm{I}=$ Imajinary part of the spectrum. $<\mathrm{X}(\mathrm{f})=$ phase spectrum of the response signal.

There is a very fast algorithm on MATLAB used to compute the Fourier transform of the response signal and phase statement is used to find the phase spectrum. In order to apply Fast Fourier transform algorithm, the response signal is sampled with sampling frequency at least equal double of the maximum frequency of the signal. The Fourier transform of the sampled signal is given by,

$X(K)=\frac{1}{\sqrt{N}} \sum_{n=0}^{N-1} x(n) e^{-j 2 \pi k n / N}$ 
Where, $\mathrm{X}(\mathrm{K})=$ Discrete frequency spectrum, $\mathrm{x}(\mathrm{n})=$ Discrete time excitation signal, $\mathrm{n}=1 \ldots \mathrm{N}-1, \mathrm{~K}=1 \ldots \mathrm{N}-1, \mathrm{~N}=$ Total number of the samples of the response signal under the window of processing. On MATLAB Fast Fourier transform algorithm is applied on Eq. 4 to determine the power spectrum, power spectrum density and phase spectrum of the exited, [9]. Cable from the following equations,

$|\mathrm{X}(\mathrm{k})|^{2}=\mathrm{R}^{2}(\mathrm{k})+\mathrm{I}^{2}(\mathrm{k})=$ Power spectrum of the response signal. $\frac{|\mathrm{X}(\mathrm{k})|^{2}}{\mathrm{~N}}=$ Power spectral density of the response signal

$-\tan ^{-1} \frac{\mathrm{I}(\mathrm{k})}{\mathrm{R}(\mathrm{k})}=$ Phase spectrum of the response signal.

Having computed the power spectrum, power spectral density and phase spectrum of the cable under test, the following relation or the frequency response is used, [5],

$\omega^{2}=\frac{\int_{0}^{1}\left(\begin{array}{c}\left(\sum_{n=1}^{\infty} B_{n}\left(\frac{2 \pi n}{1}\right) \cos \frac{2 \pi n x}{1}-\sum_{n=1}^{\infty} A_{n}\left(\frac{2 \pi n}{1}\right) \sin \frac{2 \pi n x}{1}\right)\left(\frac{m \pi}{1}\right) \frac{1}{2} \sin ^{2 m \frac{2 m \pi x}{1}}- \\ \left(A_{0}+\sum_{n=1}^{\infty} A_{n} \cos \frac{2 \pi n x}{1}+\sum_{n=1}^{\infty} B_{n} \sin \frac{2 \pi n x}{1}\right)\left(\frac{m \pi}{1}\right)^{2} \sin ^{2} \frac{m \pi x}{1}\end{array}\right) d x}{\int_{0}^{1} \rho \sin ^{2} \frac{m \pi x}{1} d x}$

Where,

$A_{o}=\frac{1}{l} \int_{0}^{l} P(x) d x$

$A_{n}=\frac{2}{l} \int_{0}^{l} P(x) \cos \frac{2 \pi n x}{l} d x=\frac{2}{l} \int_{0}^{l} \frac{P_{o}}{\left(1+C \cdot e^{\left(-\left(2 \alpha\left|x-x_{C}\right|\right) / d\right)}\right)} \cos \frac{2 \pi n x}{l} d x$

$\mathrm{B}_{\mathrm{n}}=\frac{2}{\mathrm{l}} \int_{0}^{\mathrm{l}} \mathrm{P}(\mathrm{x}) \sin \frac{2 \pi \mathrm{nx}}{\mathrm{l}} \mathrm{dx}=\frac{2}{\mathrm{l}} \int_{0}^{\mathrm{l}} \frac{\mathrm{P}_{\mathrm{o}}}{\left(1+\mathrm{C} \cdot \mathrm{e}^{\left(-\left(2 \alpha \mid x-\mathrm{x}_{\mathrm{c}}\right) / \mathrm{d}\right)}\right)} \sin \frac{2 \pi \mathrm{nx}}{\mathrm{l}} \mathrm{dx}$

$\mathrm{P}(\mathrm{x})=\sigma * \mathrm{~A}(\mathrm{x})=\frac{\mathrm{P}_{\mathrm{o}}}{\left(1+\mathrm{C} \cdot \mathrm{e}^{\left(-\left(2 \alpha\left|\mathrm{x}-\mathrm{x}_{\mathrm{c}}\right|\right) / \mathrm{d}\right)}\right)}$

And, $\mathrm{P}_{\mathrm{o}}$ : is the applied load on the cable without effect of crack, $\mathrm{C}$ : is constant equal to $\frac{A_{o}-A_{c}}{A_{c}}, A_{o}$ : is cable area without crack, $A_{c}$ : is cable area at crack section, $\mathrm{d}$ : is cable diameter, $\alpha$ : is constant equal to $0.667,[5], \mathrm{x}_{\mathrm{c}}$ : is the position of crack, $\mathrm{x}$ : is the position along the length of beam, $\sigma:$ is the applied stress of cable without effect of crack.

The variation of the above computed values for the power spectrum, power spectral density and phase spectrum are correlated with the faults on the cable to identify the cable fault. Also the analysis showed the variation of the above parameters with the location and type of the fault. Tests have been conducted for faulty and un-faulty cables and accordingly a computer program has been designed to calibrate the faults, fault location and type of the fault with variation of power spectrum, power spectral density and phase of detected response signal. The Results of the experimental tests are fed into artificial neural network (ANN) the output of ANN was found in good agreement with the experiment results of the testing the faulty and un-faulty cables. Therefore practically the output date of the experimental tests are fed directly to the ANN system to detect the faults and determine the location and type of the fault.

\section{Experimental work}

The experimental work included measuring and analyzing the exciting signal, evaluated from the accelerometer part, to three electrical-vibration characterization parts, its characterizations are power spectrum, power spectral density, and phase for excited cable. The characterizations of the vibrated cable are evaluated with various crack depths and position effect. The electrical-vibration characteristics can be evaluated by analysis the response signal of the cable by using sig-view program, [10]. Thus, the vibration test procedure for cable with various crack parametric effect is shown in Fig. 1. The values of crack depth and position are shown in Table 1. The cable is supported from both ends of the cable. Fig. 2. shows the excited cable under test with its dimensions. Thus, the electrical characteristics measured are analyzed by sig-view program into three parts as shown in Fig. 3. To verify the experimental results, they should be extracted using another technique, and the results obtained from this technique will be compared with the experimental results, to determine the percentage of error between the two applied techniques, [11-13]. ANN techniques (Artificial Neural Network) is used to evaluate the experimental, and then, both results are compared, to determine the results percentage of error between the two techniques.

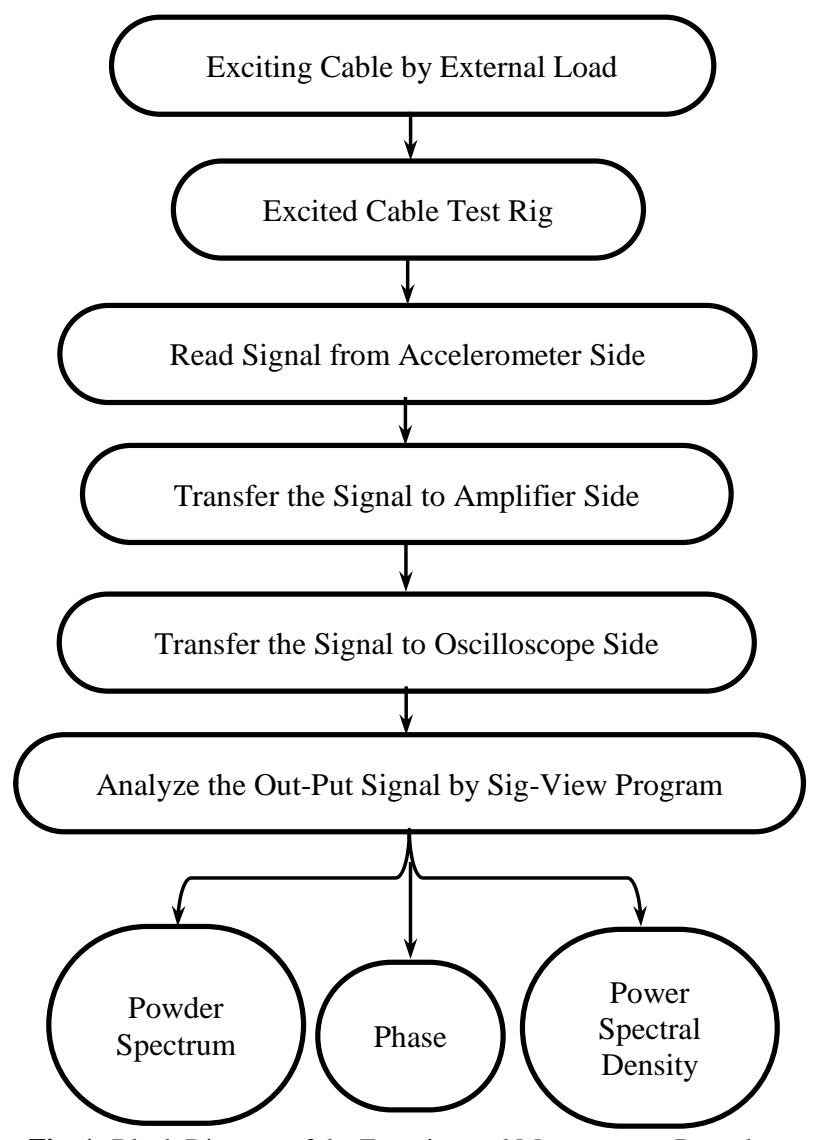

Fig. 1: Block Diagram of the Experimental Measurement Procedure

Table 1: Crack Depth and Position Used in the Experimental Work

\begin{tabular}{lll}
\hline No. & Crack Position $(\mathrm{xc} / \mathrm{L})$ & Crack Depth $(\mathrm{dc} / \mathrm{d})$ \\
\hline 1 & 0.5 & 0 to 0.9 \\
2 & 0.4 & 0 to 0.9 \\
3 & 0.3 & 0 to 0.9 \\
4 & 0.2 & 0 to 0.9 \\
5 & 0.1 & 0 to 0.9 \\
\hline
\end{tabular}

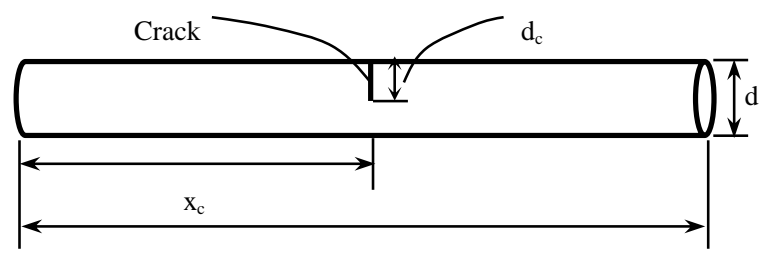

$\mathrm{L}$

Fig. 2: Typical Fiber Optical Cable Indicating the Crack.

A) Time Domain Response Signal

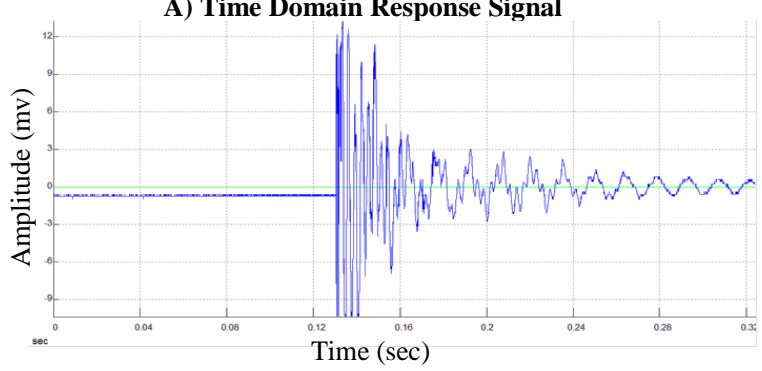


B) Frequency Domain of the Response Signal

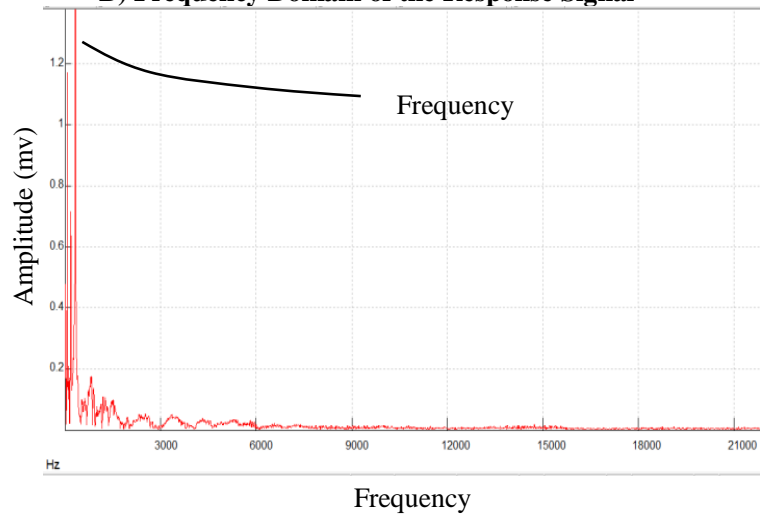

C) Power Spectrum of the Response Signal

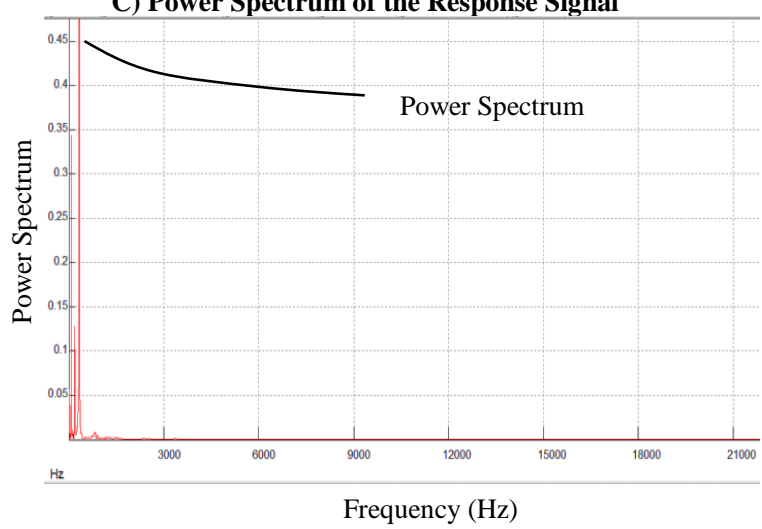

D) Power Spectral Density of the Response Signal

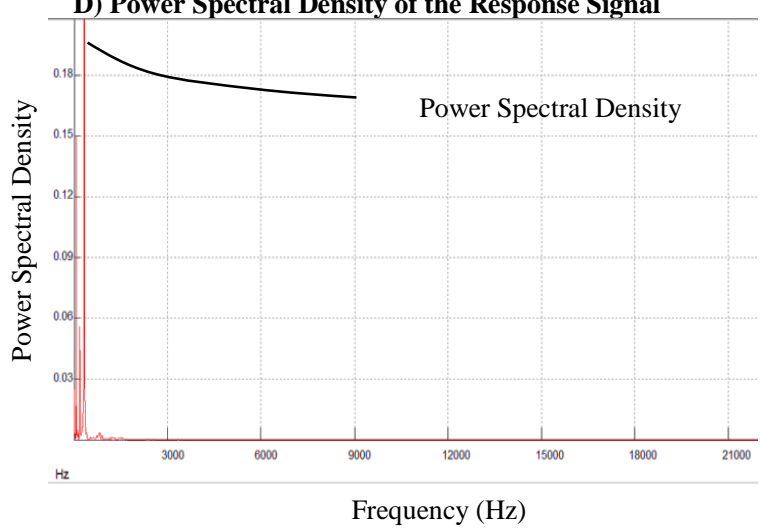

E) Phase Spectrum of the Response Signal

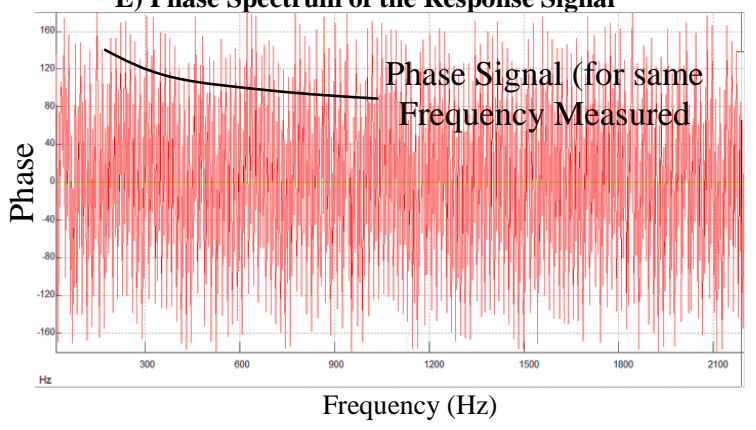

Fig. 3: Analysis of Vibration Signal by Sig-View Program.

\section{ANN technique for detection faults on fiber optical cables}

Artificial neural network (ANNS) are used in different fields of the science and technologies to solve the problems concerned with different field of the life. All the human being and animals process the information using the neural network available in their body. These networks are formed of trillions of nerve cells. These cells transfer the pulses called action software algorithms which have been designed and implemented to simulate these neurons. They are called artificial neural networks. ANN system is defined as a computing system made of many simple and highly interconnected processing elements which processes information by its dynamic state response to external input. The ANNS consists of an array of elements called neurons interconncted between them and some input/output elements, [14]. The properties of ANN system are determined by the neuron characteristic, its topology and by learning (Training) rules of neuron. The multilayer perception MLP is the most important and useful neural network in function approximation, [15]. Fig. 4. shows a typical multi-layer artificial neural network. The MLP involves input layer, many hidden layers and one output layer. The activation function used is a hyperbolic tangent (tanh) for mathematical convenience. This is defined as,

$\tanh (x)=\frac{1-e^{-x}}{1+e^{-x}}$

The output of the node I is,

$y_{i}=g_{i}=g\left(\sum_{j-1}^{k} w_{j i} \cdot x_{j}+Q_{i}\right)$

MLP is formed by connecting many node in series and parallel. The output of the system becomes,

$$
y_{i}=g\left(\sum_{j-1}^{3} w_{j i}^{2} \cdot g\left(n^{2} j_{1}\right)\right)+Q_{j 2}^{2} \approx g\left(\sum_{j-1}^{k} w_{k j}^{2} \cdot x k\left(Q_{j 1}^{2}\right)\right)+Q_{j 2}^{2}
$$

A novel multilayer ANN was implemented to detect the fault of the optical fiber cable by receiving the output of FFT process (power spectrum, power spectral density and phase) of the response signal a long the cable by using ANN techniques. The type of the algorithm used to implement the neural network in this research is Levenberg-Marquardt logarithm. The output of FFT is fed to this algorithm, all the components of ANN were created from the Simulink mat lab package. The parameters of the system are implemented in the neural software. These parameter are,

1) The response signal a long the fiber optic included the power spectral density and phase information.

2) FFT equation

3) Window function.

The software of the neural network was powered using the Mat Lab package, Fig. 5. shows the neural network software flow chart. ANN system has been designed with two layers. There are (20) neuron node in the hidden layer at the output layer there is (1) neuron node different kinds of the faults created different values of power spectral density and different phase values of the response signal have been given to the neural network. The Mat Lab commands used in the procedure are (newff, train and sim). A (MLPN) neural network, which is called (net) has been generated by mat lab command (newff) using (train) command the network training is created. All the data has been saved by these networks and trained itself on the above information. By implementing this system effectively over and optical fiber system will detect any fault on the fiber by comparing these information with the information it owns. 


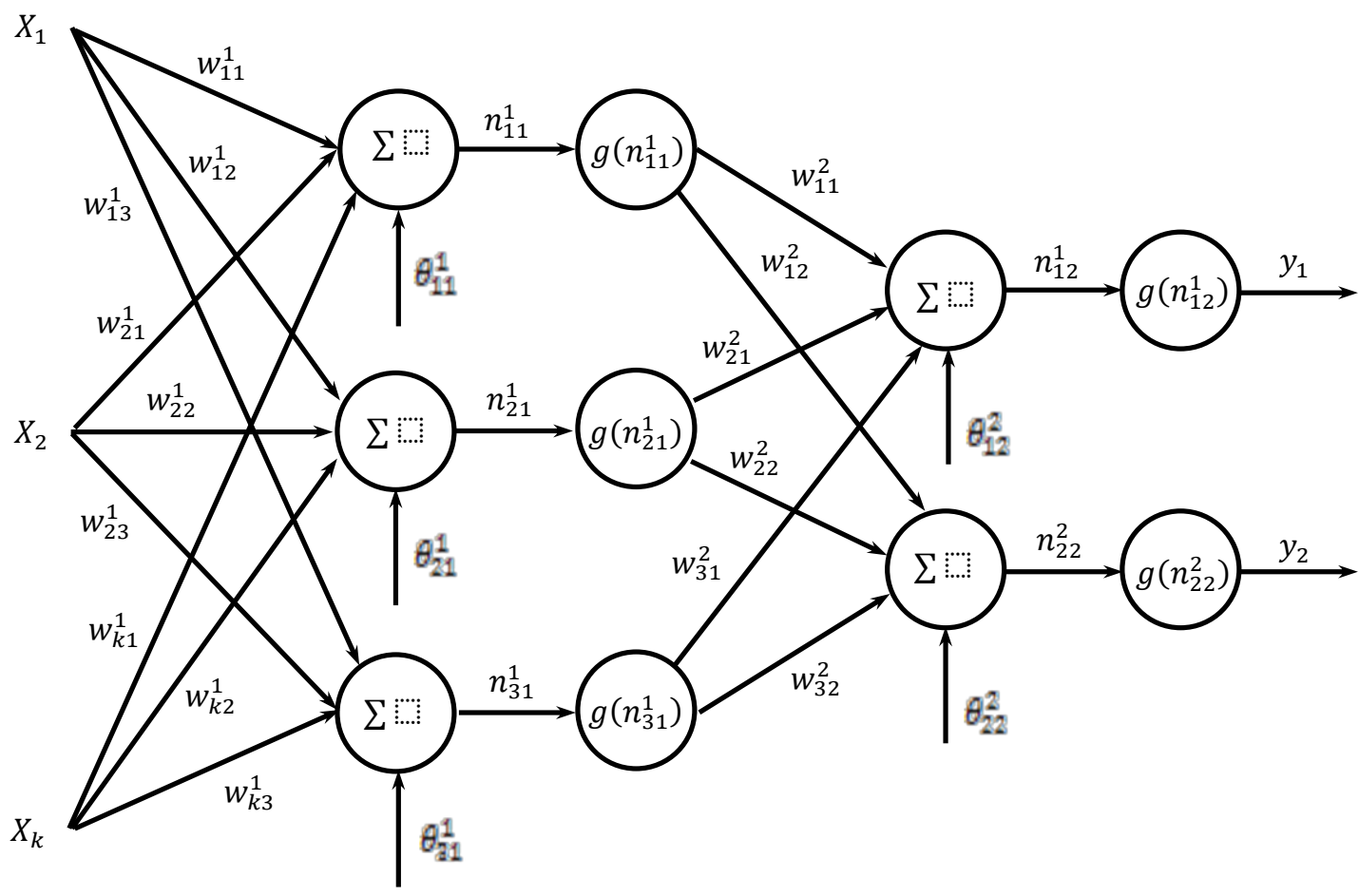

Fig. 4: A Multilayer Perceptron Network with One Hidden Layer, [15].

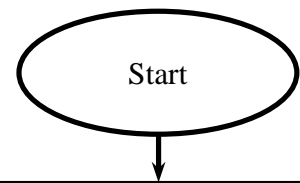

Power spectral density of the response signal from the experimental results

$>$ Power spectrum of the response signal from experimental results

$>$ Phase spectrum of the response signal from experimental results

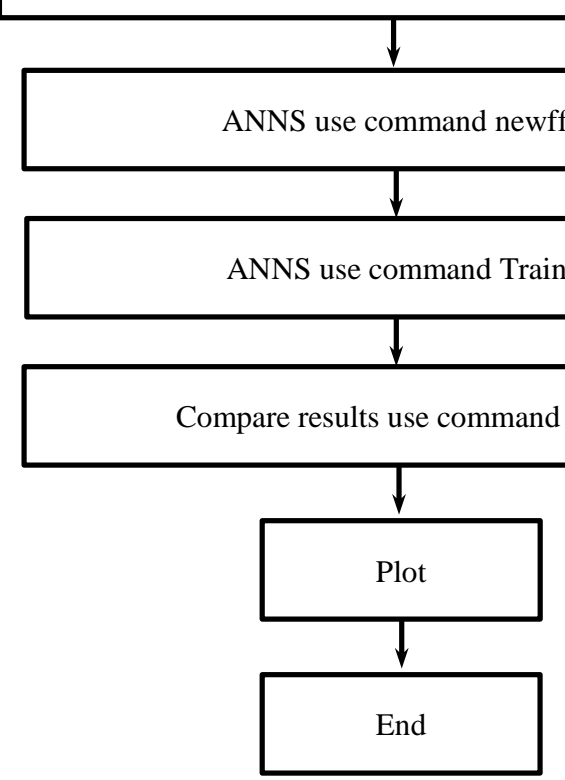

Fig. 5: Neural Network Software Flow Chart.

\section{Results and discussion}

Experimental testing of the cable for the detection of the faults on the cable has been achieved by exciting the cable with a certain vibrational signal and then detecting the response signal by a transducer. The output of the transducer has been processed and analyzed and converted to frequency domain signal by using sig-view program. This program includes FFT (Fast Fourier transform) algorithm to determine the frequency domain characteristics of the response signal, using this program the power spectrum, power spectral density and phase spectrum of the response signal were determined. The test has been repeated for faulty and un-faulty cables. Also the test was carried on with different types and positions of the faults. By using certain program, the type and position of the faulty have been identified and calibrated with determined parameter of the frequency spectrum, power spectrum, power spectral density and phase spectrum of the response signal. The output results of sigview program were applied to ANN system. Having the system been learned, the faults on the cable were detected, located and identified using this system. The output results of both experimental and ANN techniques were compared. There was a good agreement between the results of both methods. Therefore, the cable under test supported and clamped from two ends and had mechanical properties and dimensions as, [5],

1) Yield stress for cable materials $\sigma_{y}=12.1 \mathrm{Mpa}$.

2) Weight density for cable material per unit length $\rho=$ $45 \mathrm{~g} / \mathrm{m}$.

3) Diameter for cable $d=7.5 \mathrm{~mm}$.

4) Length for cable $L=0.50 \mathrm{~m}$.

\subsection{Effect of the crack on the power spectral density of the response signal}

The effect of crack depth and position on power spectral density, evaluated experimentally and using ANN techniques and results of the comparison are shown in Fig. 6. It is clear from Fig. 6. (a-e), that a good agreement between both results with maximum error about $(1.26 \%)$. The effect of crack depth and position are shown in Fig. 7. $(a, b)$ which shows that the power spectral density increases 
with increasing the crack depth, also, it increases with crack position being near the middle position of cable. From the results it is clear that the power spectral density be about $40 \%$, for crack depth $d_{c} / d=0.9$ and crack position $x_{c} / L=0.5$, of the power density of a cable without crack.

A) Crack Position $x_{c} / L=0.1$

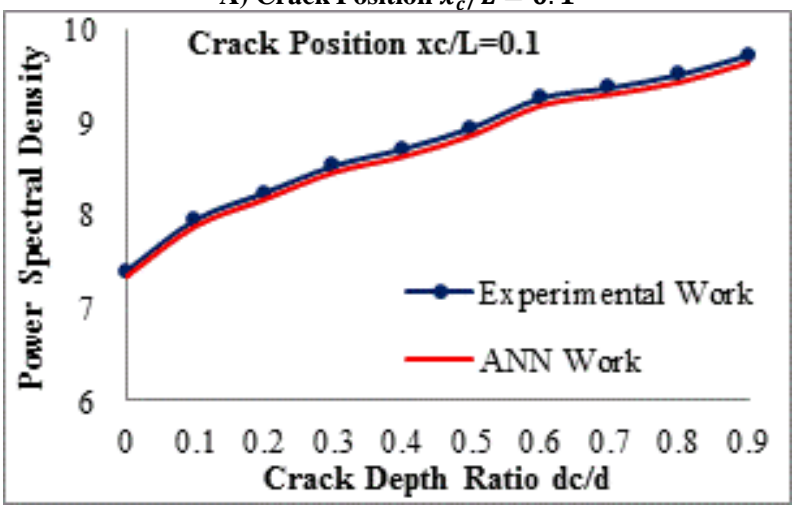

B) Crack Position $x_{c} / L=0.2$

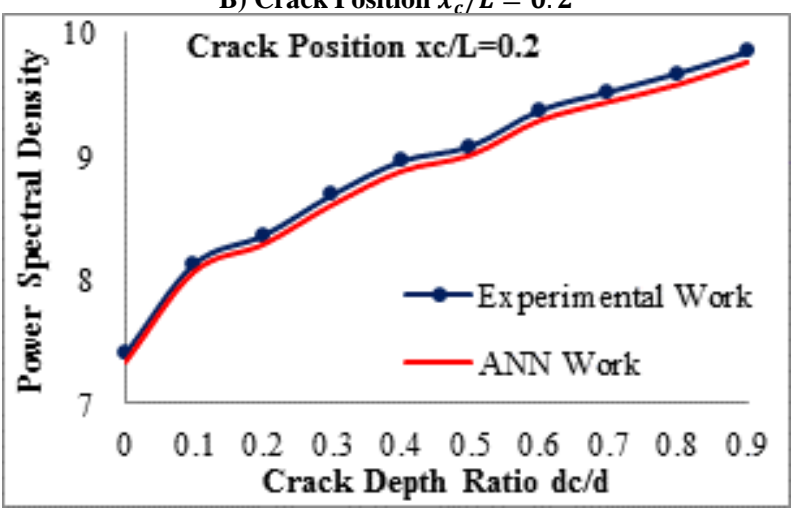

C) Crack Position $x_{c} / L=0.3$

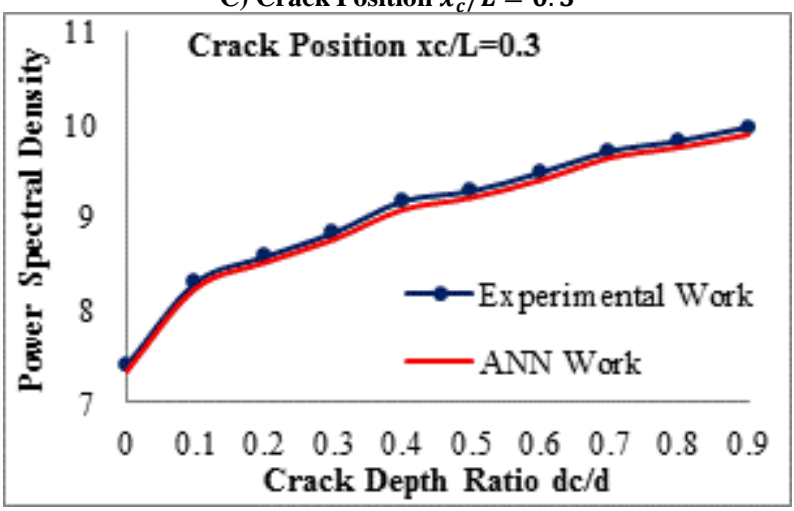

D) Crack Position $x_{c} / L=0.4$

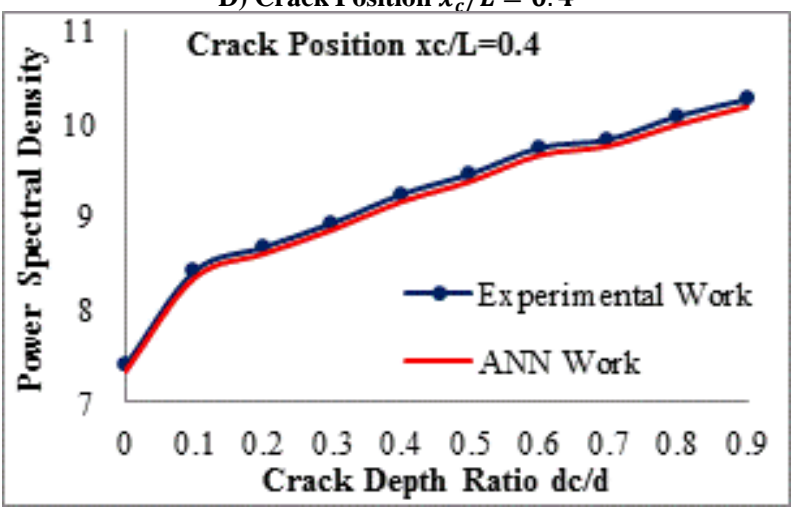

E) Crack Position $x_{c} / L=0.5$

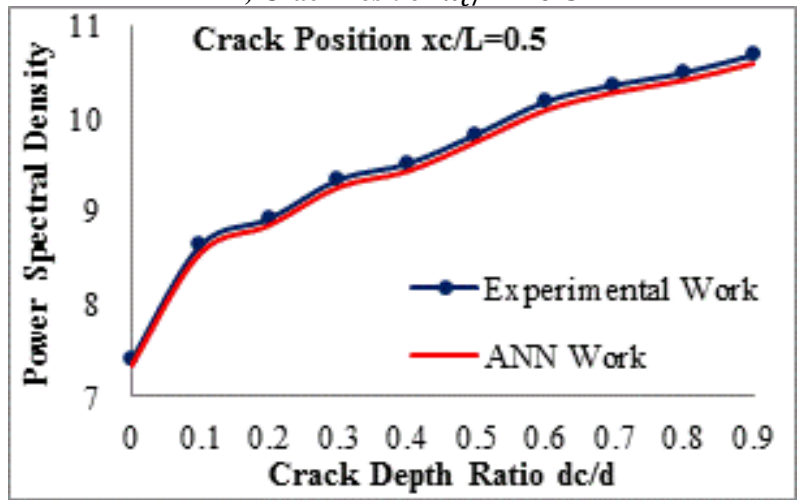

Fig. 6: Comparison between Experimental and ANN Work for Power Spectral Density Signal.

A) Crack Depth Effect.

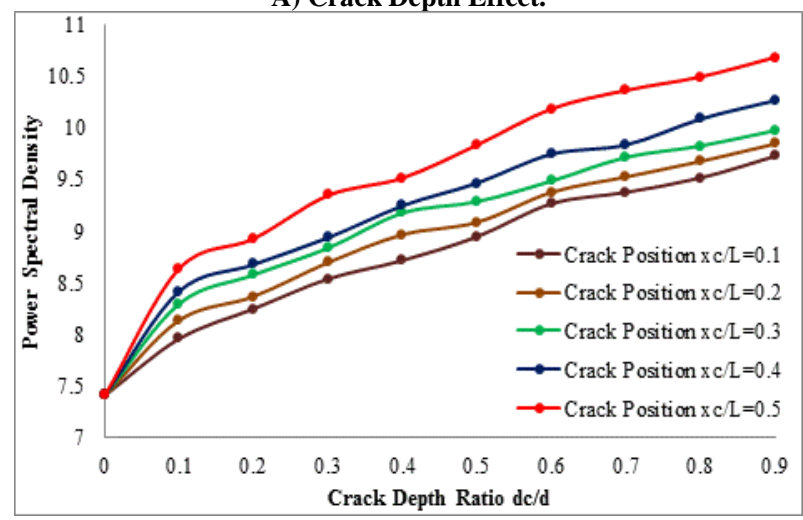

B) Crack Position Effect.

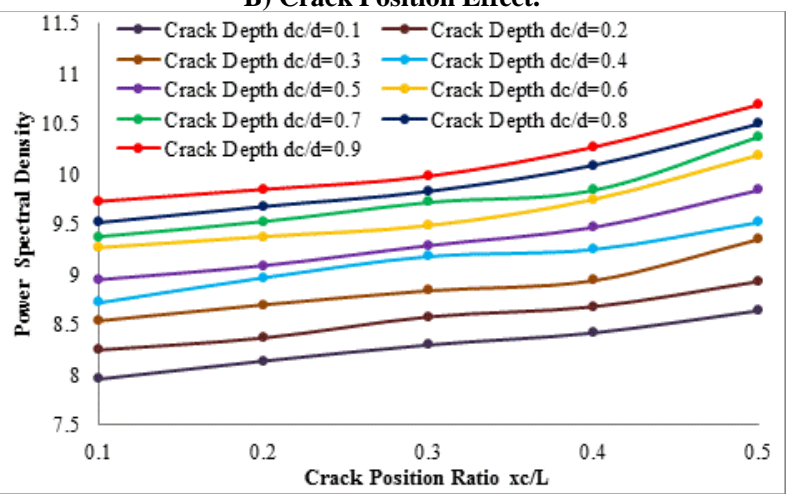

Fig. 7: Power Spectral Density for Vibrational Cable with Various Crack Depth and Position Effect.

\subsection{Effect of the crack on the power spectrum of the re- sponse signal}

The comparison between experimental and ANN results, for different crack depth and position effect, of power spectrum behavior of the cable as fixed and supported from two ends as shown in Fig. 8. (a-e) which shows that the maximum error, for results obtained by two techniques, does not exceed $1.25 \%$. The effect of crack position and depth on the power spectrum behavior is shown in Fig. 9. $(a, b)$, it is clear that the increasing of the crack depth leads to increasing the power spectrum value for the cable, in addition to, the power spectrum of the response increases with moving the crack near the middle section of the cable. 
A) Crack Position $x_{c} / L=0.1$

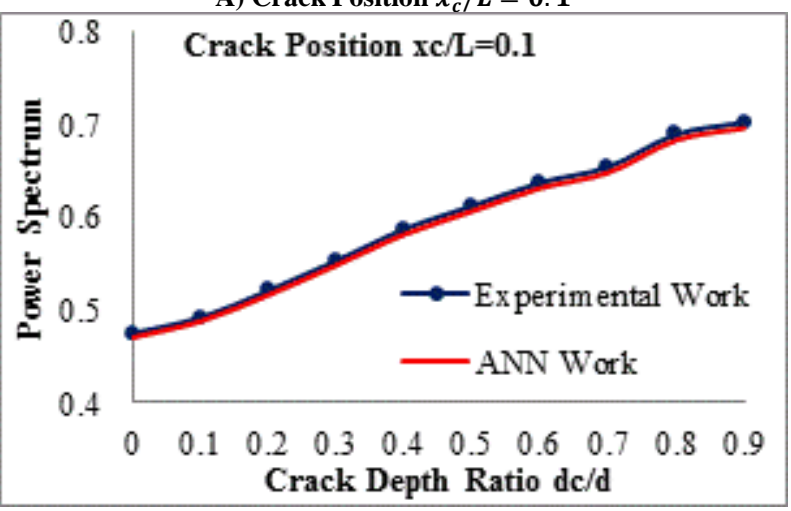

B) Crack Position $x_{c} / L=0.2$

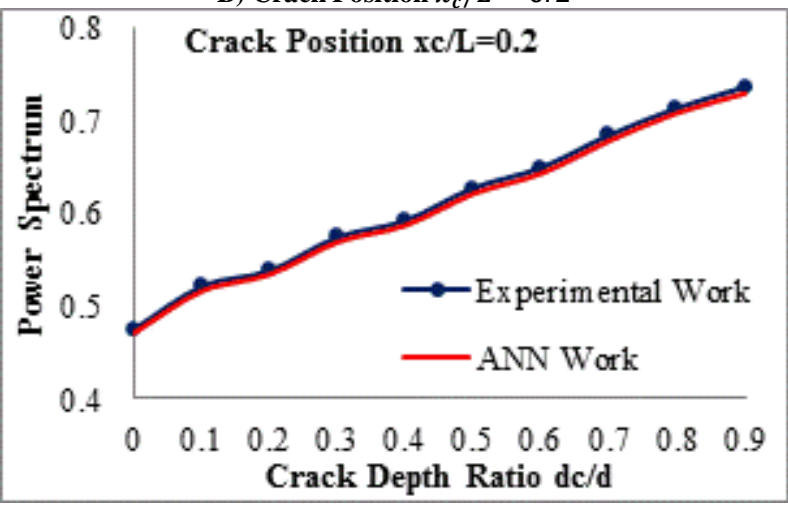

C) Crack Position $x_{c} / L=0.3$

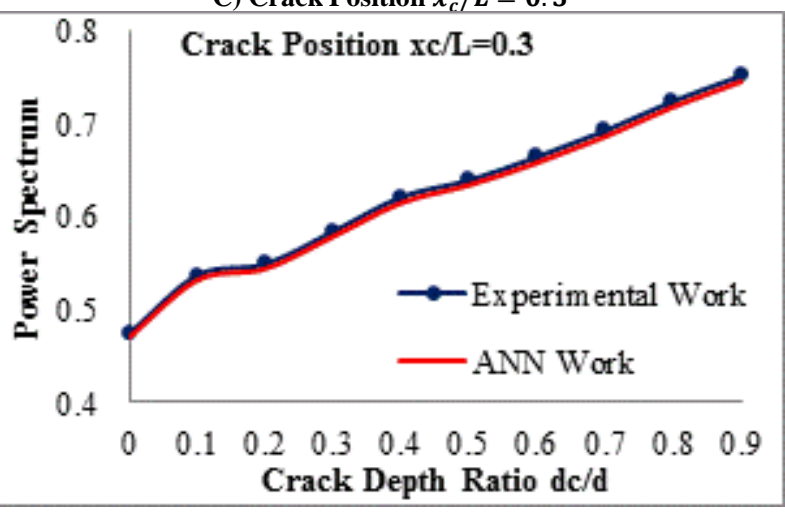

D) Crack Position $x_{c} / L=0.4$

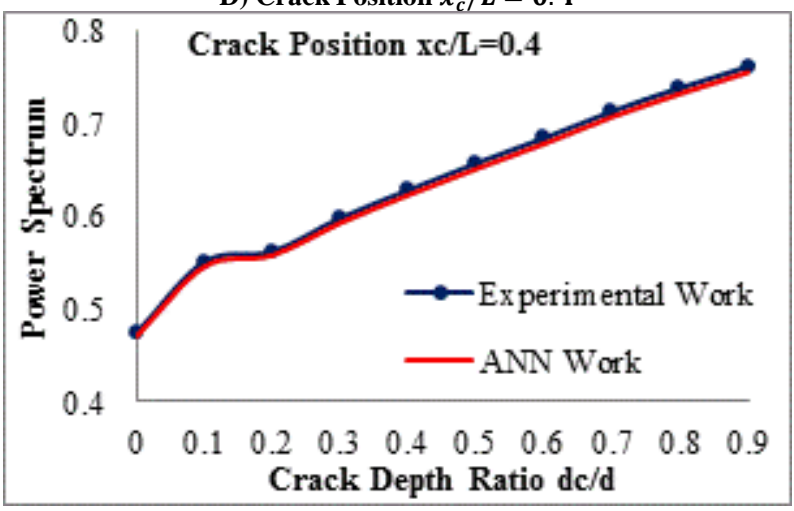

E) Crack Position $x_{c} / L=0.5$

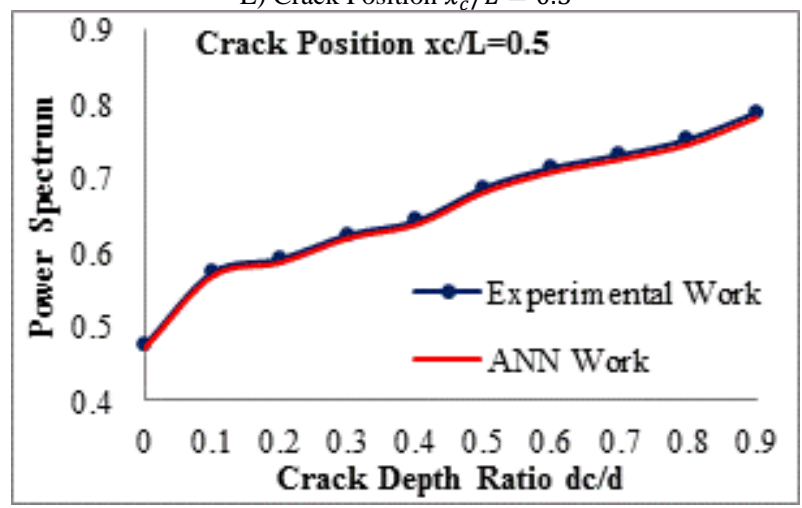

Fig. 8: Comparison between Experimental and ANN Technique for Power Spectrum of the Response Signal.

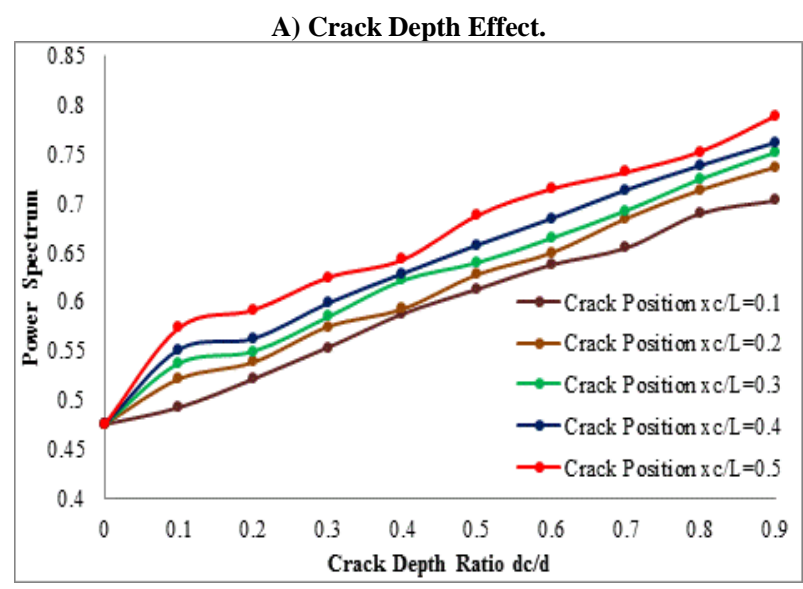

B) Crack Position Effect.

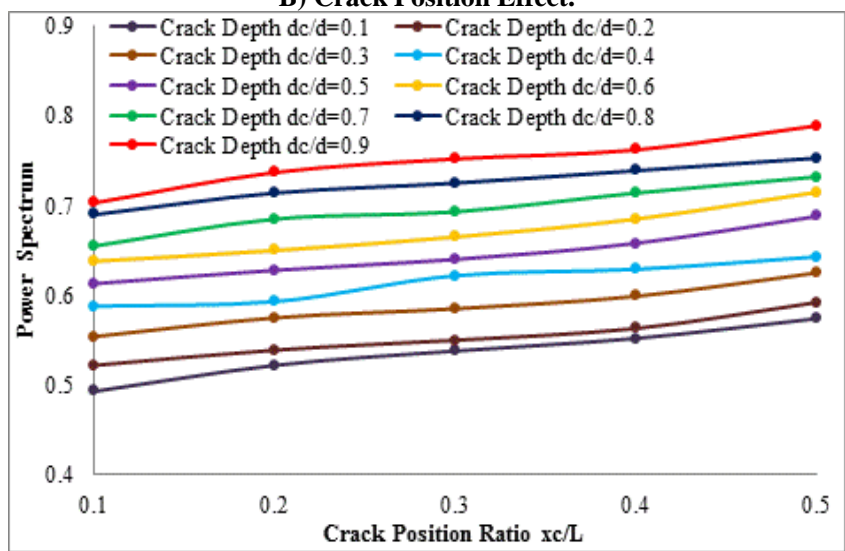

Fig. 9: Power Spectrum of the Excited Cable with Various Crack Depth and Position Effect.

\subsection{Effect of the crack on the phase spectrum of the re-} sponse signal

The phase spectrum for excited cable with crack depth and position effect was evaluated by experimental techniques, and then, compare with results that have been obtained by using ANN technique. The percentage of error of the results of both techniques is shown in Fig. 10. (a-e) which shows that the maximum error is about (1.2\%). The behavior of the phase spectrum for excited cable with various crack parameters effect are shown in Fig. 11. (a,b). It can be seen that the phase decreases with increasing the crack depth and it decreases with rounding the crack to the middle of the cable. Thus, the decrease of phase of the response of the cable under test is resulting from the decrease of the cable stiffness, due to the crack.

Finally, from Figs. 7, 9, and 11 it can be seen, due to that the crack the stiffness decreases for the cable and increases the response behavior of cable. That leads to decrease or both of power spectral 
density and power spectrum, in addition to, decrease the phase spectrum of excited cable.

A) Crack Position $x_{c} / L=0.1$

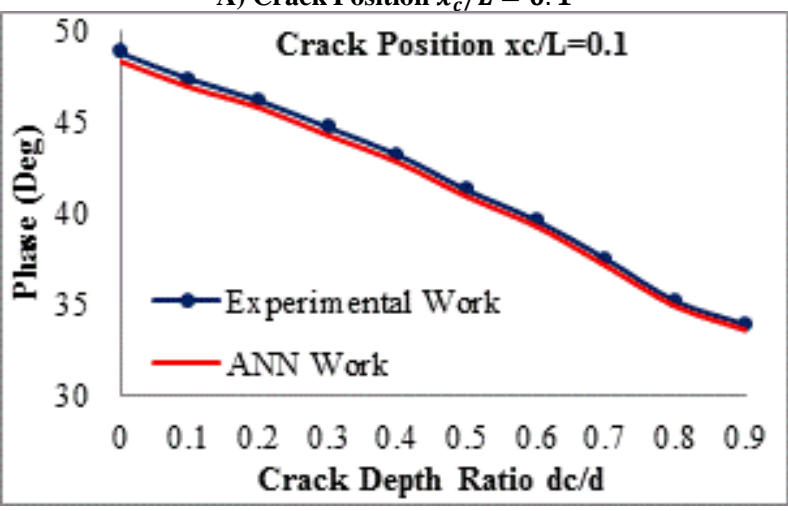

B) Crack Position $x_{c} / L=0.2$

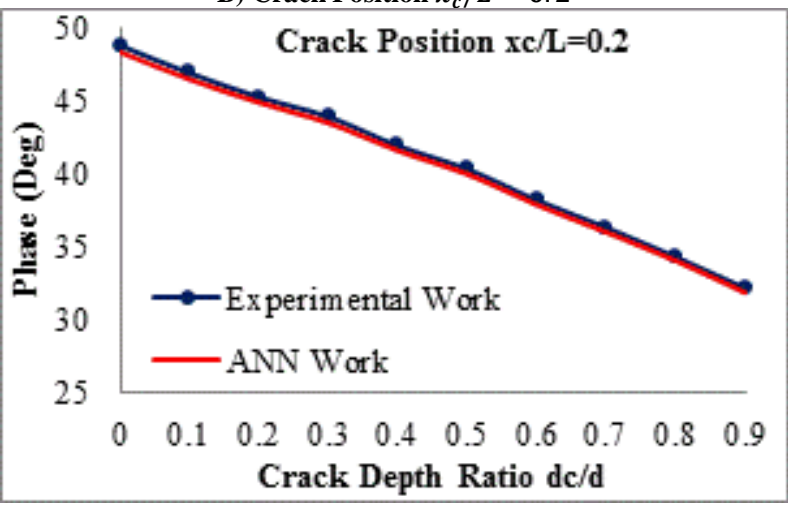

C) Crack Position $x_{c} / L=0.3$

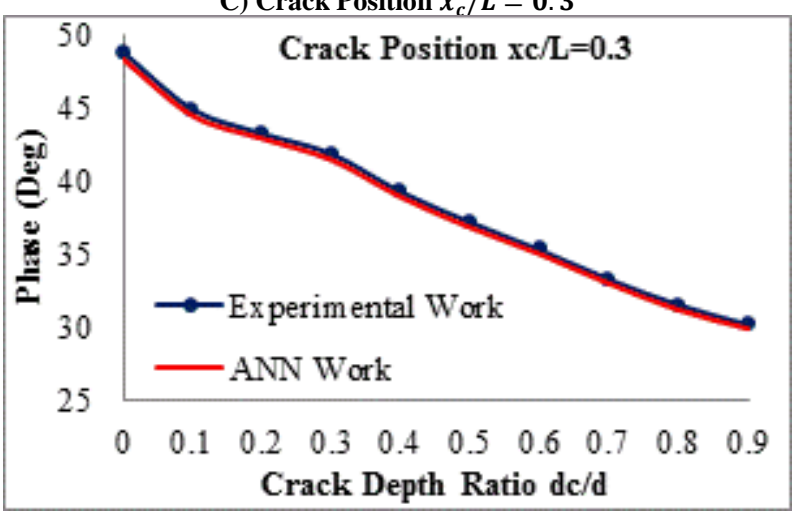

D) Crack Position $x_{c} / L=0.4$

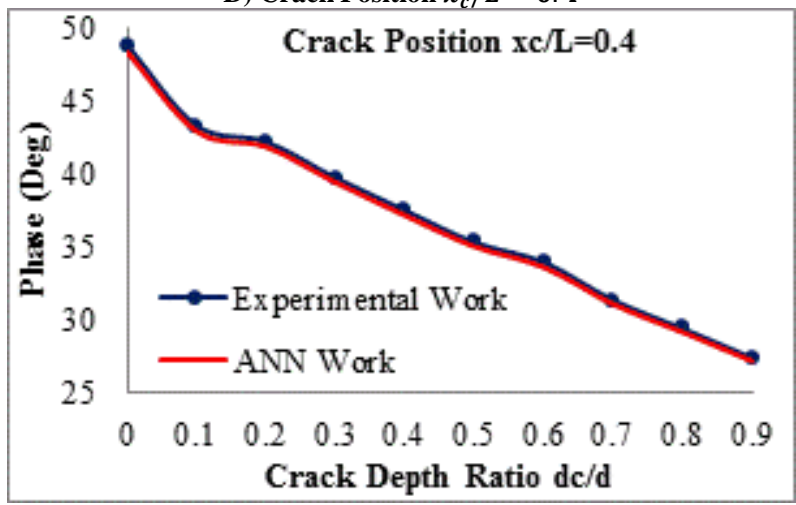

E) Crack Position $x_{c} / L=0.5$

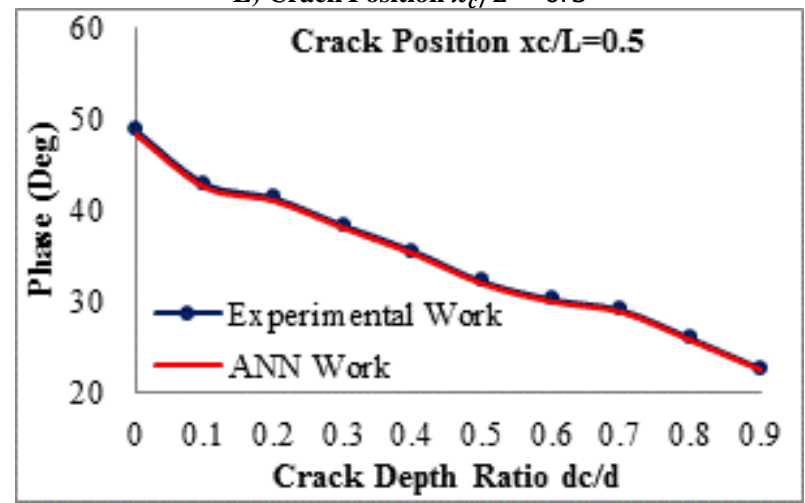

Fig. 10: Comparison Between Experimental and ANN Technique for Phase Spectrum of the Reference Signal.

A) Crack Depth Effect.

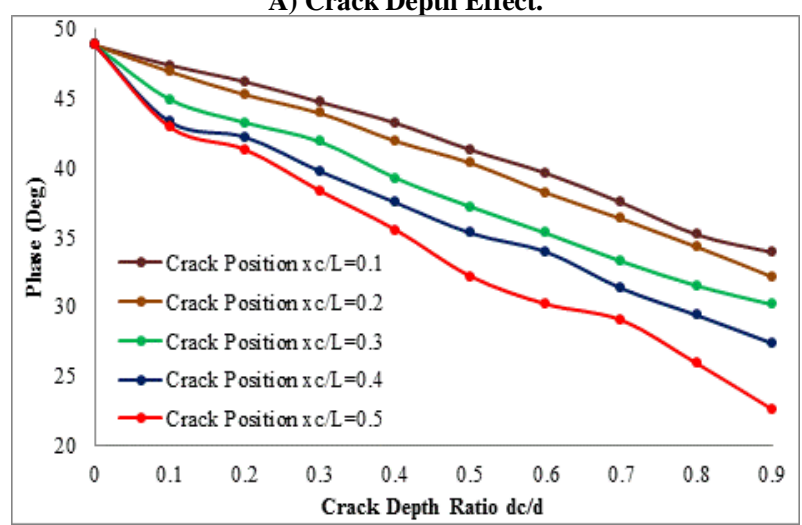

B) Crack Position Effect.

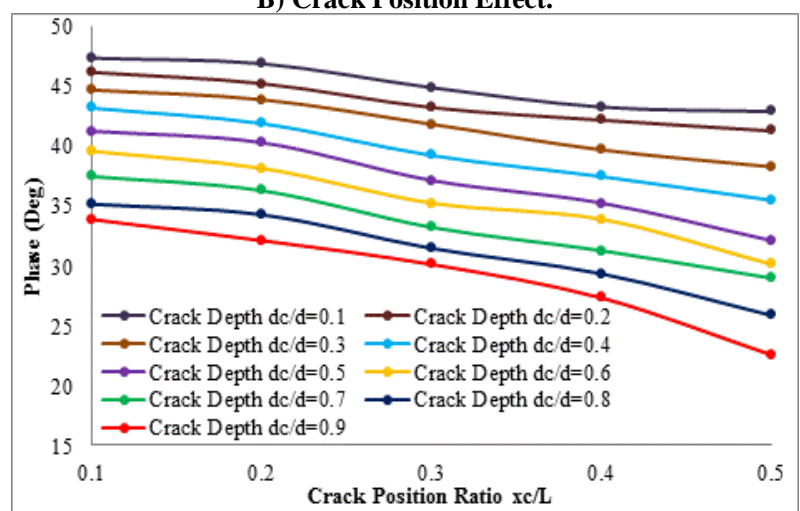

Fig. 11: Phase Spectrum of the Excited Cable with Various Crack Depth and Position Effects.

\section{Conclusion}

The investigation for crack depth and position effects on the various spectrum values, evaluated experimentally and ANN technique, has shown various important points, such as,

1) The experimental vibration technique, evaluates the spectrum characteristics of the excited cable with various crack parameters effects. It is a good technique that can be used to evaluate characteristics of the excited cable.

2) The comparison spectrum characteristics between experimental and ANN techniques has shown a good agreement results, since the maximum error between the two techniques used does not exceed about (1.26\%).

3) The power spectral density and power spectrum of the excited cable increases with increasing the crack depth and with rounding the crack to middle section of the cable under test.

4) The phase spectrum of excited cable decreases with increasing the crack depth and with rounding the crack to middle position of cable. 
5) From (power spectral density, power spectrum and phase spectrum results) the crack type and position can be predicted by analyzing the response signal response with spectrum analysis techniques using ANN system.

\section{References}

[1] Woodward W. R. 'Optical Time Domain Reflectometer-Basic Theory and Application’ W. R. System, Ltd., 2002.

[2] J. P. Von der Weid, Rogiero Passy, G. Mussi, N. Gisin 'On the Characterization of Optical Fiber Network Components with Otical Frequency Domain Reflectometry’ Journal of Lightwave Technology, Vol. 15, No. 07, pp. 1131-1141, 1997. https://doi.org/10.1109/50.596958.

[3] P. Oberson, B. Huttner, O. Guinnard, L. Guinnard, G. Ribordy, N Gisin 'Optical Frequency Domain Reflectometry with a Narrow Linewidth Fiber Laser' IEEE Photonics Technology Letters, Vol. 12, No. 07, pp. 867-869, 2000. https://doi.org/10.1109/68.853529.

[4] Jihong Geng, C. Spiegelberg, Shibin Jiang 'Narrow Linewidth Fiber Laser for 100-km Optical Frequency Domain Reflectometry' IEEE Photonics Technology Letters, Vol. 17, No. 09, pp. 1827-1829, 2005. https://doi.org/10.1109/LPT.2005.853258.

[5] Hussain. J. Abbas, Muhsin. J. Jweeg, Muhannad Al-Waily, Abbas Ali Diwan 'Experimental Testing and Theoretical Prediction of Fiber Optical Cable for Fault Detection and Identification' Journal of Engineering and Applied Sciences, Medwell Journals, Accepted to Publication, Vol. 13, 2018.

[6] Muhsin J. Jweeg, Ali S. Hammood, Muhannad Al-Waily 'A Suggested Analytical Solution of Isotropic Composite Plate with Crack Effect' International Journal of Mechanical \& Mechatronics Engineering IJMME-IJENS, Vol. 12, No. 05, 2012.

[7] Muhannad Al-Waily, Maher A. R. Sadiq Al-Baghdadi, Rasha Hayder Al-Khayat 'Flow Velocity and Crack Angle Effect on Vibration and Flow Characterization for Pipe Induce Vibration' International Journal of Mechanical \& Mechatronics Engineering IJMME-IJENS, Vol.17, No.5, 2017.

[8] Steven V. W. 'Digital Signal Processing" 2nd Edition, California, Technology, pp. 225-415, 1999.

[9] Burak K. 'Practical Usage of Fast Fourier Transform (FFT)' AMSC Document Information, Vol. 0, 2004

[10] Muhannad Al-Waily, Kadhim K. Resan, Ali Hammoudi Al-Wazir, Zaman Abud Almalik Abud Ali 'Influences of Glass and Carbon Powder Reinforcement on the Vibration Response and Characterization of an Isotropic Hyper Composite Materials Plate Structure' International Journal of Mechanical \& Mechatronics Engineering IJMME-IJENS, Vol.17, No.6, 2017.

[11] Muhsin J. Jweeg, S. Z. Said 'Effect of Rotational and Geometric Stiffness Matrices on Dynamic Stresses and Deformations of Rotating Blades' Journal of the Institution of Engineers, Mechanical Engineering Division, India, Vol. 76, May 1995.

[12] Mohsin Abdullah Al-Shammari, Muhannad Al-Waily 'Theoretica and Numerical Vibration Investigation Study of Orthotropic Hyper Composite Plate Structure' International Journal of Mechanical \& Mechatronics Engineering IJMME-IJENS, Vol. 14, No. 6, 2014

[13] Muhsin J. Jweeg, Muhannad Al-Waily, Alaa Abdulzahra Deli 'Theoretical and Numerical Investigation of Buckling of Orthotropic Hyper Composite Plates' International Journal of Mechanical \& Mechatronics Engineering IJMME-IJENS, Vol.15, No. 4, 2015.

[14] David J. Cavuto 'An Exploration and Development of Current Artificial Neural Network Theory and Applications with Emphasis on Artificial Life' M.Sc. Thesis, the Cooper Union, Albert Nerken School of Engineering, 1997.

[15] Heikki N. Koivo 'Neural Networks: Basics using MATLAB Neural Network Toolbox' Heikki Koivo, 2008 